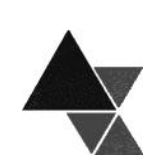

\title{
Adesão às refeições oferecidas pelo Programa Nacional de Alimentação Escolar em uma cidade do sudoeste do Paraná
}

\author{
Camila Elizandra Rossi ${ }^{1}$, Franciane Silvana Formentini ${ }^{2}$, Áline Mayara Ferreira ${ }^{3}$
}

Avaliou-se a execução do Programa Nacional de Alimentação Escolar em município do Sudoeste do Paraná. Tratou-se de estudo quanti-qualitativo, descritivo, realizado em junho de 2011, em 7 escolas de ensino fundamental da rede pública (urbanas e rurais). Analisou-se a adesão ao programa em oito dias não consecutivos, em cada escola, definindo-a como o ato do estudante de se servir da alimentação escolar. Calculou-se o Índice de Atendimento Efetivo (\%IAE = total de alunos atendidos/total de alunos presentes na escola $\mathrm{x} 100$ ), classificando-o em: alta (acima de 70\%), média (50 a 70\%), baixa (30 a 50\%) e muito baixa adesão (> 30\%). Foram entrevistados atores envolvidos na gestão e na execução do programa, incluindo-se nutricionista responsável técnica na época, o coordenador administrativo do programa e um servidor do setor de Vigilância Sanitária. As entrevistas foram feitas com questionário aberto e semiestruturado. Observou-se alta adesão $(75,2 \%)$ nas 56 refeições avaliadas e alta adesão/escola em 71,42\% das mesmas. A adesão mais alta foi em escola da zona rural. Ao analisar cada escola, somente 1 teve todas as preparações com alta adesão, também na zona rural. Preparações completas foram ofertadas em sua maioria na zona rural, e, nas urbanas, lanches e sobremesas. As entrevistas mostraram fragilidades no Conselho de Alimentação Escolar e variedade expressiva de frutas, legumes e verduras adquiridos da agricultura familiar.

Palavras-chave: alimentação escolar, adesão ao programa, políticas públicas.

\section{Food adhesion in the School Feeding Brazilian Program in a municipality in the South}

We aimed to evaluate the execution of the National School Feeding Program (Brazil), in a small city of the Southern of Paraná State (South of Brazil). This study was quanti-qualitative and descriptive, realized in July 2011, with the seven public schools of the municipality (rural and urban areas). Adhesion was evaluated in eight nonconsecutive days, in each school, and was defined as the student's attitude of serving themselves of school meals. Index adhesion was calculated taking account the Index of Effective Attendance (IEA $\%=$ total number of scholars attended/number of scholars in class x 100), and classified how: high (above 70\%), medium (between 50 to $70 \%$ ), low (30 to 50\%) and very low adhesion (>30\%). We interviewed the staff involved with the technical responsibility and management of the program, including a nutritionist, an administrator and a person of the sanitary vigilance. Interviews were made using an open semistrutured questionnaire. We found a high adhesion among the 56 meals served (75.2\%), and too high adhesion/school in $71.42 \%$ of them. The mayor adhesion was observed in one school of the rural zone. But, when each meal was observed just 1 school had high adhesion in all meals, and this was one of the rural schools. Complete meals were offered more frequently in

\footnotetext{
1 Professora do Curso de Nutrição, Universidade Federal da Fronteira Sul. Correspondência: Rua Zelindro Périco no 2.191. CEP: 85770-000, Realeza, Paraná, Brasil. Telefone: (46) 9935-4317. E-mail: camilarossi@uffs.edu.br

2 Acadêmica do Curso de Nutrição da Universidade Federal da Fronteira Sul. E-mail: francianeformentini@hotmail.com

${ }^{3}$ Acadêmica do Curso de Nutrição da Universidade Federal da Fronteira Sul. E-mail: mayaferreira1@gmail.com
} 
rural zone, while sweet ones and deserts were offered in urban schools. Interviews showed fragilities in the Food Council and an expressive variety in fruits and vegetables which are acquired from familiar agriculture.

Key-words: school feeding, adhesion to the program, public policies.

\section{INTRODUÇÃO}

Políticas públicas são importantes ferramentas para combater distúrbios nutricionais, e nessa perspectiva merece destaque o Programa Nacional de Alimentação Escolar (PNAE) [1], programa embasado na formação de hábitos alimentares saudáveis de escolares em toda a rede pública de educação básica brasileira [2].

O PNAE integra a política de Segurança Alimentar e Nutricional (SAN), definida pela Lei no 11.346, de 16 de setembro de 2006, em seu Art. 3o, como "a realização do direito de todos ao acesso regular e permanente a alimentos de qualidade, em quantidade suficiente, sem comprometer o acesso a outras necessidades essenciais, tendo como base práticas alimentares promotoras de saúde que respeitem a diversidade cultural e sejam ambientais, cultural, econômica e socialmente sustentável". Essa lei encarrega o poder público de adotar as ações e políticas que se façam necessárias, a fim de promover e garantir segurança alimentar e nutricional da população e $\mathrm{O}$ Direito Humano à Alimentação Adequada [3].

O PNAE é orientado pela Lei no 11.947/2009 e pela Resolução no 26/MEC/CD/2013, do Fundo Nacional de Desenvolvimento e Educação (FNDE), que preveem a oferta de uma alimentação de qualidade, como direito de todos os estudantes [2,4], também assegurado por meio do Art. 208 da Constituição da República Federativa do Brasil [5]. A resolução, em seu Art. $2^{\circ}$, determina que a alimentação escolar seja direito dos alunos da educação básica pública e dever do Estado, e traz como diretriz o emprego da alimentação saudável e adequada, compreendendo o uso de alimentos variados, que respeitem a cultura e tradição local, e prevê ainda a inclusão da educação alimentar nutricional no processo de ensino e aprendizagem [2].

A mesma resolução traz, em sua III diretriz, Art. 2o, "a universalidade do atendimento aos alunos matriculados na rede pública de educação básica" [2]. Para assegurar que a universalidade do atendimento do PNAE aos escolares seja efetiva, a adesão dos beneficiados da alimentação escolar constitui um dos recursos para verificar se as preparações são as mais adequadas possíveis à população a que está destinada.

Segundo Sturion [6], é importante investigar a adesão e registrar os resultados, pois estes auxiliam e apontam a necessidade de reavaliação das preparações distribuídas pelo Programa de Alimentação Escolar, visando à incorporação, na medida do possível, de alimentos/preparações nutricionalmente balanceadas e que atendam às preferências dos alunos.

Segundo a Resolução no 26/MEC/CD/2013, o programa tem como objetivo contribuir para $O$ crescimento e desenvolvimento biopsicossocial, a aprendizagem e rendimento escolar, bem como a formação de práticas alimentares saudáveis por meio de ações de educação nutricional, ao mesmo tempo em que cobre as necessidades nutricionais dos alunos durante o período letivo [2]. Tendo conhecimento da importância da alimentação escolar para a melhor aprendizagem, o rendimento escolar e a formação de hábitos saudáveis, bem como a segurança alimentar e nutricional no ambiente escolar, destaca-se, portanto, a importância desta pesquisa que teve por objetivo avaliar a execução do PNAE e a adesão das preparações servidas para os escolares, no município de Realeza, localizado no sudoeste do estado do Paraná, verificando assim a atuação e desenvolvimento do programa em nível local.

\section{MÉTODOS}

Trata-se de subprojeto de uma pesquisa de maior abrangência intitulada "Análise do Programa Nacional de Alimentação Escolar e do perfil nutricional de estudantes do ensino fundamental", realizada com escolares de 1 ao 5o ano da rede pública de um município do Sudoeste do Paraná.

Esta pesquisa é de caráter quanti-qualitativo, realizada em junho de 2011, em 7 escolas da rede pública, incluindo-se urbanas e rurais. 
Adesão foi definida, neste estudo, como o ato do estudante de se servir da alimentação escolar, independente de ingerir. Para avaliá-la, foi realizada a contagem do número de escolares presentes e o número dos que se serviram da alimentação oferecida em cada escola $(n=7)$, durante 8 dias não consecutivos. Com esses dados, calculou-se o percentual de adesão, utilizando-se a fórmula do Índice de Atendimento Efetivo - IAE (\%IAE=total de alunos atendidos/total de alunos presentes na escola $x$ 100), conforme proposto por Gandra e Gambardella [7], com auxílio do programa Calc do Br-Office $e^{\circledR}$ para tabular os dados.

A classificação do índice de adesão dos escolares ao programa realizou-se por meio do sugerido pelo Centro Colaborador em Alimentação e Nutrição do Escolar - CECANE - da UNIFESP [8], o qual referenda a utilização dos pontos de corte definidos por Sturion [9], considerando-se quatro categorias: alta adesão (acima de 70\%), média adesão (50 a 70\%), baixa adesão (30 a 50\%) e muito baixa adesão (menos que $30 \%)$.

Para avaliar outros parâmetros da execução do PNAE, foram realizadas entrevistas com a nutricionista responsável técnica pelo programa, o gestor administrativo e uma fiscal da vigilância sanitária. Foi adotado formulário semiestruturado, com perguntas abertas, previamente testado em estudo piloto. Os questionamentos versaram sobre o tipo de alimentos adquiridos, se convencionais ou orgânicos, instrumentos de compra, percentual de recursos investidos na Agricultura Familiar e presença destes produtos no cardápio das unidades educativas, atuação do Conselho de Alimentação Escolar (CAE) e controle de qualidade.

Esta pesquisa foi aprovada pelo Comitê de Ética em Pesquisa da Faculdade Assis Gurgacz (FAG) por meio do protocolo no $11 / 2011$.

\section{RESULTADOS E DISCUSSÃO}

Ao avaliar-se a adesão geral nas sete escolas do município, observou-se que aproximadamente três quartos $(75,2 \%)$ dos escolares se serviram da alimentação oferecida. Encontrou-se, portanto, alta adesão, como também observado por Bleil et al. ${ }^{[10]}$ no município de Toledo - PR (oeste do Paraná), onde se observou proporção de adesão à alimentação escolar de $77,3 \%$. Estes índices são esperados pelos gestores e responsáveis técnicos do programa e podem ser reflexo de contínuos esforços para a melhoria da execução do mesmo, desde a sua criação. Especialmente após a década de 1990, com a descentralização da compra dos alimentos e com a aquisição de gêneros da agricultura familiar a partir de 2009, produtos in natura e itens condizentes com os hábitos alimentares dos escolares puderam ser inseridos [11]. Outra possível explicação para a alta adesão é a ausência de cantinas escolares em algumas escolas de Realeza. Leme, Philippi e Toassa [12], em sua pesquisa com adolescentes de uma escola de São Paulo - SP para saber se preferiam os alimentos da escola ou os competitivos, verificaram que muitos consumiam alimentos na escola por ser a única alternativa durante o intervalo de aula, devido à inexistência de cantina.

Resultados contraditórios foram encontrados por Danelon et al. [13], em estudo da adesão ao programa em Campinas - SP, em que verificaram (mediante o questionamento se os escolares consumiam ou não as refeições) baixa adesão, pois apenas 38,3\% dos alunos se alimentavam entre quatro e cinco dias por semana (frequência semanal considerada efetiva adesão pelo estudo). Os demais aderiam com menor frequência ao programa: três vezes por semana $(15,7 \%$ ), ou raramente a consumiam (entre um e dois dias por semana $-21 \%$ ).

Santos et al. [14], avaliando a adesão ao programa em Pelotas - RS observaram que, do total de alunos entrevistados, 95,6\% $(\mathrm{n}=86) \quad$ afirmaram consumir a alimentação oferecida pela escola, sendo que $61,1 \%$ relataram consumir entre $4-5$ vezes na semana, se enquadrando em uma adesão média. $\mathrm{O}$ fator que possivelmente reduziu a adesão, segundo os autores, foi o consumo de outro tipo de alimentação que não a escolar, ainda que a escola não possuísse cantina, pois o hábito de trazer alimentos de casa foi citado por $52,2 \%(n=47)$ dos estudantes. Quanto aos lanches trazidos de casa, no momento da coleta de dados em Realeza, os pesquisadores perceberam que poucos estudantes tinham consigo alimentos diferentes daqueles servidos no cardápio escolar, o que propicia a adesão.

Em relação às diferenças de adesão entre as cidades, ao se consultar o censo demográfico por município, é possível perceber que Realeza contava com cerca de 16.338 habitantes, enquanto Pelotas e Campinas, com mais de 328 mil e mais de 1 milhão de habitantes, respectivamente [15]. Ou seja, parece que quanto maior o porte da cidade, menor é a adesão à alimentação distribuída na escola. Em cidades menores, é possível que a quantidade de estabelecimentos que 
comercializem alimentos seja menor, e, quanto a redes de fast e junk food, praticamente inexistentes, ao contrário do que se encontra em metrópoles. Ball, Timpério e Crawford [16] já observaram que as escolhas alimentares, desde meados da década de 1990, têm sido modificadas pelo ambiente no qual as populações vivem e, portanto, a exposição a estabelecimentos que comercializam alimentos pode propiciar a menor adesão à refeição servida na escola, pois há mais oportunidade de acessar alimentos fora de casa e da escola. Estudos sobre a densidade de estabelecimentos comerciais em cidades (ambiente alimentar) e sua relação com a adesão ao PNAE poderiam elucidar esta possível relação.

No presente estudo, ao se analisar a adesão à alimentação entre as escolas rurais e urbanas, notou-se que a adesão ao programa foi maior na zona rural
(80,4\% versus 70,0\%). Não foram avaliados os fatores que possam ter levado a uma maior adesão nas escolas rurais, mas é possível que se relacione à menor renda nessas localidades. Hoffmann [17] objetivando avaliar os efeitos de diversos fatores na probabilidade de um aluno consumir a alimentação servida na escola, em unidades de ensino fundamental público, verificou que a probabilidade de consumo das refeições foi maior para alunos da área rural e menor com o aumento da renda.

Em relação ao tipo de refeições ofertadas, avaliou-se, no presente estudo, a frequência de preparações doces e salgadas em cada escola, dentre o total de 8 refeições avaliadas em cada uma delas (Tabela 1).

Tabela 1. Frequência das refeições oferecidas quanto ao tipo de preparação, em uma cidade do sudoeste do Paraná 2011.

\begin{tabular}{l|c|c|c|c|c|c|c}
\hline \multirow{2}{*}{ Escola } & \multirow{2}{*}{$\begin{array}{c}\text { Localidade } \\
\text { da escola }\end{array}$} & \multicolumn{3}{|c|}{ Tipo de preparação } & \multicolumn{3}{c}{ Classes de preparações } \\
\cline { 3 - 8 } & Salgada & Doce & Completa & Lanche & Sobremesa & $\begin{array}{c}\text { Somente } \\
\text { frutas }\end{array}$ \\
\hline Escola 1 & Urbana & 2 & 6 & 2 & 4 & 2 & - \\
Escola 2 & Urbana & 5 & 3 & 5 & 3 & - & - \\
Escola 3 & Rural & 8 & - & 7 & 1 & - & - \\
Escola 4 & Rural & 5 & 3 & 4 & 3 & 1 & - \\
Escola 5 & Urbana & 1 & 7 & - & 6 & 2 & - \\
Escola 6 & Urbana & 1 & 7 & - & 5 & 3 & 3 \\
Escola 7 & Urbana & 4 & 4 & 3 & 2 & - & - \\
\hline
\end{tabular}

Observou-se que nas escolas rurais prevaleceu a oferta de preparações salgadas, em sua maioria compostas de refeições completas (incluindo carne ou ovos, saladas, frutas e/ou legumes e carboidratos complexos), como, por exemplo, arroz, feijão, carne moída, beterraba e laranja. Verificou-se, ainda, que uma das escolas rurais serviu oito preparações salgadas $(100 \%)$, e $87,5 \%(n=7)$ delas eram completas. Já nas escolas urbanas, a prevalência maior foi de preparações doces. Somente em uma escola urbana observou-se prevalência de $62,5 \%$ de preparações salgadas $(n=5)$, sendo que em duas escolas urbanas (a mais próxima do centro da cidade) houve prevalência de $87,5 \%$ de preparações doces. Em outro estudo de
Danelon [18], percebeu-se que, em muitos municípios, é frequente a oferta de preparações salgadas completas, as quais foram mais oferecidas em escolas localizadas em bairros de baixo nível socioeconômico, que costumam se localizar em regiões não centrais. Ainda, como as preparações doces também tiveram boa adesão em uma das escolas rurais, é possível que os escolares dessa área precisem de um aporte energético maior (devido à insegurança alimentar, à distância casa-escola, ou ao fato de terem um gasto maior de energia). Já os alunos das escolas urbanas podem ter maior nível de segurança alimentar e nutricional, preferirem preparações do tipo lanche em vez de grandes refeições e também porque o município oferta 
atividades escolares em período integral (servindo almoço ao meio-dia) em algumas escolas urbanas. A respeito da segurança alimentar e nutricional, dados recentes da Pesquisa Nacional por Amostras de Domicílio (PNAD) revelaram que a insegurança alimentar nacional continua mais prevalente na zona rural que na urbana $(13,9 \%$ versus $6,8 \%)$, e não houve melhora nos índices de segurança alimentar neste local entre os anos de 2004 e 2013 (64,8\% para 64,7\%) [19], o que talvez possa explicar os dados encontrados neste estudo.

Pôde-se ainda observar na Tabela 1 que apenas uma escola ofereceu, em 3 dos dias analisados, preparações compostas somente por frutas, ou preparações em que estas eram o ingrediente principal. As demais escolas não tiveram nenhuma preparação composta somente por frutas, apesar de estarem inseridas em algumas preparações como sobremesa. Por exemplo, nas preparações consideradas completas, foi comum a presença da laranja. Já algumas preparações classificadas como lanches traziam as frutas na sua formulação, como vitamina de banana, ou como acréscimo ao lanche principal, como foi o caso do cachorro-quente com maçã. Nestes dias, a adesão foi próxima da considerada boa. Apesar disso, servir refeições compostas apenas por frutas é algo a se planejar com cautela, visto que a Resolução no 26/MEC/CD/2013 [2] prevê a quantidade de nutrientes a serem ofertados por faixa etária, os quais não são atingidos ao se oferecer refeições compostas somente por frutas, legumes ou verduras. Isso é especialmente importante de se observar em escolas de regiões com vulnerabilidade social.

Os pesquisadores observaram também que algumas escolas urbanas ofereceram suco artificial junto a algumas preparações completas. A Resolução no 26/MEC/CD/2013 [2] traz em suas disposições a proibição da compra de bebidas de baixo valor nutricional, tais como refrigerantes e refrescos artificiais, com recursos do FNDE. A mesma proibição já estava registrada na resolução de 2009 [20], em vigor no ano da coleta dos dados do presente estudo. Nas escolas rurais, a única bebida servida foi o chá, aparecendo duas vezes em cada escola. Verificouse, ainda, que em $100 \%$ das escolas urbanas houve a oferta da preparação sucrilhos (cereal de milho) misturado a leite, flan de chocolate ou creme, alguns acompanhados de frutas in natura. Nas escolas rurais não foi observada a oferta da preparação cereal de milho. Dentre estas preparações, a que teve menor percentual de adesão foi sopa de legumes, com
$28,47 \%$ em uma das escolas analisadas. Outra escola também teve a mesma preparação analisada, com adesão de 60,24\%. Em contrapartida, a preparação que obteve maior adesão foi o cachorro-quente, acompanhado de chá, ou fruta in natura, obtendo $100 \%$ de adesão em 50\% das escolas onde foi servido. A preparação cachorro-quente também foi citada por Elert et al. [21] que encontraram como alimentos/preparações preferidos mais citados pelos alunos: feijão $(32,4 \%)$, bolacha $(31,1 \%)$, creme de chocolate $(31,1 \%)$, arroz doce com leite $(29,7 \%)$, cachorro-quente $(29,7 \%)$, leite $(28,4 \%)$ e arroz (25,7\%). Leme, Philippi e Toassa [12], ao entrevistarem os alunos da escola pública de São Paulo, observaram que $38,5 \%$ consomem as refeições e o pão com salsicha é citado como um dos pratos servidos na escola. Sobre isso, ressalta-se que a Resolução no 26/MEC/CD/2013 [2] traz em suas disposições a restrição da compra de alimentos ricos em sódio, tais como embutidos, com recursos do FNDE.

Martins et al. [22] também observaram diferenças no percentual de adesão ao programa de acordo com o tipo de preparação oferecida. O estudo envolveu 12 unidades de ensino do município de Piracicaba (São Paulo), verificando que a adesão às preparações servidas no horário do intervalo das atividades escolares foi baixa ( $40 \%$ a $50 \%$ ), exceto para preparações como macarronada ou arroz com molho à bolonhesa, com proporção acima de 50\% (indicação de adesão classificada como média).

Sturion et al. [6], em estudo de nível nacional realizado em dez municípios brasileiros no ano de 2002, encontraram que a baixa adesão à alimentação escolar deveu-se a alguns fatores, tais como: preparações inadequadas aos horários de distribuição, temperatura inadequada e não atendimento das preferências dos escolares, entre outros, sendo que alguns desses fatores podem ser minimizados com adequações na gestão do programa. Esses fatos podem estar relacionados com a reduzida adesão encontrada para a preparação sopa de legumes que foi servida ao final do mês de setembro em Realeza, quando as temperaturas já começavam a aumentar e motivavam as preferências por preparações frias. Desde o ano pesquisado até a atualidade, portanto, é possível perceber que ainda melhorias na oferta das refeições podem ser realizadas. Apesar dos avanços na gestão nacional do programa [11], parece que as fragilidades em nível local, tais como qualidade sensorial, uso de utensílios adequados, cardápios adaptados ao clima, presença de refeitórios, ainda 
carecem de solução, persistindo as mesmas ao longo dos anos.

Analisando a adesão média de cada escola, segundo a classificação proposta por Sturion [i], 71,4\% das mesmas apresentaram alta adesão e 28,6\% média.
Contudo, ao analisar cada preparação, somente 14,3\% $(\mathrm{n}=1)$ das escolas teve todas as preparações com alta adesão, e esta escola era de área rural (Escola 4) Tabela 2.

Tabela 2. Adesão média das preparações servidas no PNAE, de acordo com as escolas, em uma cidade do sudoeste do Paraná - 2011.

\begin{tabular}{cc}
\hline Escola / Localização & Adesão (\%) \\
\hline Escola 1 (Urbana) & 68,90 \\
Escola 2 (Urbana) & 63,18 \\
Escola 3 (Rural) & 74,05 \\
Escola 4 (Rural) & 87,71 \\
Escola 5 (Urbana) & 78,50 \\
Escola 6 (Urbana) & 78,66 \\
Escola 7 (Urbana) & 75,60 \\
\hline
\end{tabular}

Segundo a classificação proposta por Sturion [9], 64\% $(n=36)$ das preparações oferecidas em todas as escolas analisadas obtiveram alta adesão, 32\% $(\mathrm{n}=18)$ média adesão, somente uma preparação (pão com doce de leite, banana e suco de uva de pacote) obteve adesão baixa, e também uma (sopa de legumes) teve adesão muito baixa (Figura 1). As duas preparações classificadas como baixa e muito baixa adesão foram servidas na mesma escola (Escola 2), situada na área urbana da cidade.

Figura 1. Índice de efetiva adesão das preparações, em uma cidade do sudoeste do Paraná - 2011.

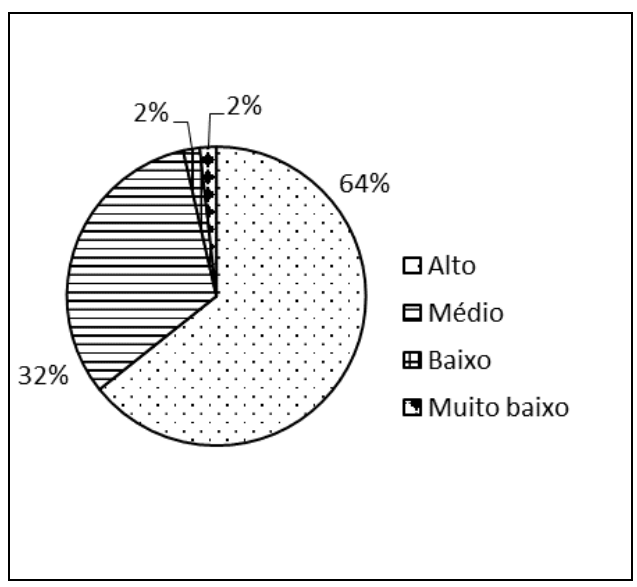


A adesão a cada preparação pode ter se relacionado a fatores como: forma de distribuição dos alimentos, incorporação dos hábitos alimentares locais, ambiente físico, utensilios, higiene dos mesmos, e as pessoas que assumem papel junto à alimentação escolar, incluindo nutricionista responsável técnica e as cozinheiras, conforme observaram Teo et al. [23], em estudo realizado em Chapecó - SC. Neste mesmo estudo, a presença de refeitórios, autosserviço, menor presença de cantinas, desenvolvimento de ações de educação nutricional, exposição de materiais educativos, e incorporação de verduras e legumes aos cardápios nas escolas municipais fizeram com que a adesão fosse maior, quando comparada a escolas estaduais [23].

No município paranaense avaliado, ao coletar os dados, os pesquisadores observaram que algumas preparações poderiam ter tido melhor aparência e ser servidas em utensílios em melhor estado de conservação. Em algumas escolas não havia refeitório, o que pode ter diminuído a adesão nas mesmas. Observou-se também que a relação merendeiraescolares pode ter interferido no fato de o aluno aderir ou não ao alimento por ela preparado. Os fatores citados pelos demais estudos e as percepções descritas poderão ser alvo de trabalhos futuros que confirmem estes pressupostos.

Ainda, a relação destes fatores com a alimentação escolar de Realeza fica evidente, visto que a vulnerabilidade social não é tão expressiva no município. Dados do Instituto Paranaense de Desenvolvimento Econômico e Social (IPARDES), publicados no ano de 2013, como Índice de Desenvolvimento Humano Municipal (IDH-M) e Índice de Gini (IG) se mostraram favoráveis. O IDH foi de 0,722 e o IG foi de 0,438 [24]. O Programa das Nações Unidas para o Desenvolvimento (PNUD) considera desenvolvimento médio quando os valores de IDH ficam entre 0,500 e 0,799 [25]. Já os índices de Gini mais próximos de zero mostram melhor distribuição de renda entre a população, sendo que valores abaixo de 0,500 são avaliados como ideais.

Quanto ao cardápio da alimentação escolar para o ano avaliado, tinha potencial para ser atrativo e adaptado aos hábitos locais, visto que por meio da entrevista com a nutricionista pôde-se constatar que os alimentos utilizados eram variados, contando com 23 itens entre frutas, legumes e verduras. Esses alimentos provinham todos da agricultura familiar.
A respeito da aquisição de orgânicos, as falas revelaram desconhecimento sobre o modo de produção dos hortifrutis adquiridos da agricultura familiar, mostrando pouco envolvimento entre os setores. Já o setor de Vigilância Sanitária, mostrou-se mais presente, visto que relatou realizar visitas periódicas a estes fornecedores, os quais estavam gradativamente atendendo às exigências legais. As falas sobre a compra de orgânicos, ainda, explicitou falta de intenção na compra dos mesmos, o que depõe contra os princípios da sustentabilidade ambiental e da saúde inerentes ao conceito de segurança alimentar e nutricional.

A respeito dos instrumentos de compra, foi citada a licitação para a aquisição dos mercados locais. Mas, apesar das falas indicarem uma compra separada da licitação para a agricultura familiar, não foi citada a chamada pública.

A respeito do percentual de recursos investidos na Agricultura Familiar, os entrevistados citaram que são gastos $60 \%$ dos recursos neste setor. Não ficou claro, no entanto, se esse montante era relativo aos recursos provindos do FNDE ou da contrapartida municipal. Isso pode mostrar uma falta de aproximação com a nova legislação do programa, que tinha dois anos de publicação quando das entrevistas. Comparando-se as entrevistas da nutricionista e do gestor notaram-se opiniões diferentes sobre a aquisição dos alimentos oriundos da agricultura familiar. A nutricionista relatou seu receio em precisar de alguns alimentos-base e os mesmos não serem plantados ou produzidos pelos agricultores familiares, pois algumas demandas não tinham sido cumpridas. Mesmo assim, a opinião da profissional sobre a parceria era de que esses alimentos têm qualidade e que a iniciativa é importante aos agricultores devido à questão social. Já o gestor, independente da garantia da entrega dos alimentos e da qualidade, citou pretender continuar comprando os alimentos da agricultura familiar, até atingir um valor de 100\% de aquisição, apesar de algumas experiências prévias de não-cumprimento do contrato. Ambos os entrevistados, portanto, acreditavam na possível organização do setor para um fornecimento regular e constante.

Sobre a atuação do CAE, houve diferentes opiniões e uma contradição. A nutricionista citou que as reuniões não vinham sendo realizadas e que acreditava que a nutricionista anterior a ela, integrava o conselho. Já o gestor, acreditava que o CAE estava 
atuante, que as reuniões eram semestrais, pois não havia "necessidade de chamar mais vezes os membros do conselho". E, acreditava que a nutricionista anterior não fazia parte do conselho. Observou-se, ainda, juízo de pouca importância à instância, pois foi chamada de "reuniãozinha semestral", por um dos entrevistados.

Frente a estes resultados, vê-se que é importante mais que oferecer uma alimentação para sanar a fome, visto que esse não parece ser um problema expressivo no município. É preciso atender às necessidades nutricionais, características sensoriais e hábitos alimentares dos escolares, independente de sua renda, pois quanto mais estes quesitos forem adequados, mais provável será a adesão e a aceitabilidade da alimentação escolar. E, desta forma, tanto a universalização do programa, prevista em seu segundo princípio ${ }^{[3]}$, quanto a segurança alimentar e nutricional dos escolares serão atendidas.

\section{CONCLUSÃO}

No presente estudo, em que se objetivou avaliar a adesão dos escolares ao PNAE, foi encontrada adesão alta na maioria das escolas analisadas.

Também foi possível verificar os diferentes tipos de preparações oferecidas nas localidades urbana e rural, sendo que as preparações completas foram mais frequentemente ofertas na zona rural e também neste local obtiveram maior adesão. Futuros estudos que elucidem os motivos das diferenças na oferta e na adesão ao programa entre zona urbana e rural são necessários. Variáveis como a renda e a posse de bens de consumo dos familiares, que possibilitam categorizar as famílias em classes econômicas, assim como a aplicação da Escala Brasileira de Insegurança Alimentar (EBIA) poderiam elucidar as razões para estes achados.

As entrevistas revelaram um município com potencial para compra da agricultura familiar, porém, com necessidade de esclarecimentos técnicos sobre a execução de alguns pontos relativos às finanças do programa. Mostrou também que os orgânicos ainda não são um objetivo no município. Sobre o CAE, cabe uma formação para a equipe, a fim de que possa ser sensibilizada para a importância da participação social nas políticas públicas.
Destaca-se, por fim, o importante papel da escola como uma ferramenta essencial para motivar a formação de bons hábitos alimentares, por meio da educação alimentar e nutricional e das refeições ofertadas, e consequentemente proporcionar a Segurança Alimentar neste ambiente.

\section{REFERÊNCIAS}

[1] Danelon MAS, Danelon MS, Silva MV. Serviços de alimentação destinados ao público escolar: análise de convivência do Programa de Alimentação Escolar e das cantinas. Segur Aliment Nutr. 2006;13(1):85-94.

[2] Ministério da Educação, Fundo Nacional de Desenvolvimento da Educação, Conselho Deliberativo (BR). Resolução no 26, de 17 de junho de 2013. Dispõe sobre o atendimento da alimentação escolar aos alunos da educação básica no âmbito do Programa Nacional de Alimentação Escolar - PNAE. Diário Oficial da União, 17 jun 2013.

[3] Brasil. Lei no 11.346, de 16 de setembro de 2006. Cria o Sistema Nacional de Segurança Alimentar e Nutricional SISAN com vistas em assegurar o direito humano à alimentação adequada e dá outras providências. Diário Oficial da União, 18 set 2006.

[4] Brasil. Lei no 11.947, de 16 de junho de 2009. Dispõe sobre o atendimento da alimentação escolar e do Programa Dinheiro Direto na Escola aos alunos da educação básica; altera as Leis nos 10.880, de 9 de junho de 2004, 11.273, de 6 de fevereiro de 2006, 11.507, de 20 de julho de 2007; revoga dispositivos da Medida Provisória no 2.178-36, de 24 de agosto de 2001, e a Lei no 8.913, de 12 de julho de 1994; e dá outras providências. Diário Oficial da União, 16 jun 2009.

[5] Brasil. Constituição (1988). Constituição da República Federativa do Brasil. Brasilia, DF, 5 out. 1998.

[6] Sturion GL, Silva MV, Ometto AMH, Fortuoso COM, Pipitone MAP. Fatores condicionantes da adesão dos alunos ao Programa de Alimentação Escolar no Brasil. Rev Nutr. 2005;18(2):167-81.

[7] Gandra JR, Gambardella AMD, editores. Avaliação de serviços de alimentação e nutrição. São Paulo: Sarvier; 1983. $116 \mathrm{p}$.

[8] Centro Colaborador em Alimentação e Nutrição Escolar (BR). Manual para aplicação dos testes de aceitabilidade no Programa Nacional de Alimentação Escolar - PNAE. Santos: Universidade Federal de São Paulo; 2010. 56 p. 
[9] Sturion GL. Programa de Alimentação Escolar: avaliação do desempenho em dez municípios brasileiros. Campinas (SP) [tese]. Campinas: Universidade Estadual de Campinas; 2002.

[10] Bleil RAT, Salay E, Silva MV. Adesão ao Programa de Alimentação Escolar por Alunos de Instituições Públicas de Ensino no Município de Toledo, PR. Segurança Alimentar e Nutricional. 2009; 16(1): 65-82.

[11] Peixinho AML. A trajetória do Programa Nacional de Alimentação Escolar no período de 2003-2010: relato do gestor nacional. Ciência e Saúde Coletiva. 2013;18(4):909-16.

[12] Leme ACB, Philippi ST, Toassa EC. O que os adolescentes preferem: os alimentos da escola ou os alimentos competitivos? Saúde Soc. 2013; 22(2):456-67.

[13] Danelon MS, Fonseca MCP, Silva MV. Preferências alimentares no ambiente escolar. Segurança Alimentar e Nutricional. 2008; 15(2): 66-84.

14] Santos AP, Pinheiro CT, Maciel FV, Geiger JM, Ramires MCC. Adesão ao programa nacional de alimentação escolar por estudantes de uma escola municipal de Pelotas-RS. In: II Congresso de Iniciação Cientifica e II mostra Cientifica [Internet]; 2010 nov. 9-11; Pelotas: Universidade Federal de Pelotas; 2010 Disponível em: http://www2.ufpel.edu.br/cic/2010/cd/pdf/CS/CS_0116 4.pdf

[15] Instituto Brasileiro de Geografia e Estatística - IBGE: Cidades [Internet]; 2014. Censo Demográfico 2010: sinopse [acesso 26 fev 2015]. Disponível em: http://cidades.ibge.gov.br

[16] Ball K, Timpério AF, Crawford DA. Understanding environmental influences on nutrition and physical activity behaviors: where should we look and what should we count? [Internet]. Inter J Behav Nutr Phys Act. 2006;3(33): Disponível em: http:/ /www.ijbnpa.org/content/3/1/33

[17] Hoffmann R. Determinantes do consumo da merenda escolar no Brasil: análise dos dados da PNAD de 2004 e 2006. Segur Aliment Nutr. 2012;19(1):33-45.

[18] Danelon, MAS. Modelos de gestão do Programa de Alimentação Escolar: a experiência aplicada às unidades de tempo integral [dissertação]. Piracicaba: Universidade de São Paulo; 2007.
[19] Instituto Brasileiro de Geografia e Estatística. Pesquisa Nacional por Amostra de Domicilios: segurança alimentar 2013. Rio de Janeiro: Instituto Brasileiro de Geografia e Estatística; 2014. 123 p.

[20] Ministério da Educação, Fundo Nacional de Desenvolvimento da Educação, Conselho Deliberativo (BR). Resolução no 38, de 16 de julho de 2009. Dispõe sobre o atendimento da alimentação escolar aos alunos da educação básica no Programa Nacional de Alimentação Escolar - PNAE. Diário Oficial da União, 16 jul 2009; Seção V.

[21] Elert VW, Cunha GK, Fernandes MP, Peter NB, Leal CMA. Avaliação da adesão ao Programa de Alimentação Escolar por alunos de séries iniciais de uma escola municipal de Pelotas (RS) In: XX congresso de iniciação cientifica e II mostra Cientifica [Internet]. 2011 nov 8-11; Pelotas: Universidade Federal de Pelotas; 2011. Disponível em: http://www2.ufpel.edu.br/cic/2011/anais/pdf/CS/CS_01 596.pdf

[22] Martins RCB, Medeiros M, Ragonha GM, Olbi JH, Segatti M, Osele MR. Aceitabilidade da alimentação escolar no ensino público fundamental. Saude Rev. 2004;6(13):71-8.

[23] Teo CRPA, Correa EM, Gallina LS, Fransozi C. Programa nacional de alimentação escolar: adesão, aceitação e condições de distribuição de alimentação na escola. Nutrire. 2009;34(3):165-85.

[24] Instituto Paranaense de Desenvolvimento Econômico e Social - IPARDES: perfil avançado dos municípios [Internet]. Curitiba: 2011. Perfil avançado do município de Realeza [acesso 26 fev 2015]. Disponível em: http://www.ipardes.gov.br/perfil_municipal

[25] Programa das Nações Unidas para o Desenvolvimento. Relatório de desenvolvimento humano 2007/2008: combater as alterações climáticas: solidariedade humana num mundo dividido. Coimbra, PT: Instituto Português de Apoio ao Desenvolvimento; 2007 [acesso 25 ago 2008]. 386 p. Disponível em http://www.pnud.org.br/HDR/arquivos/RDHglobais/hdr 2007-8-portuguese.pdf 